\title{
Spectroscopic study of the possibly triaxial transitional nucleus ${ }^{75} \mathrm{Ge}$
}

\section{Y. Niu (牛晨阳), ${ }^{1}$ A. C. Dai (戴阿灿), ${ }^{1}$ C. Xu (徐川), ${ }^{1,}{ }^{*}$ H. Hua (华辉), ${ }^{1, \dagger}$ S. Q. Zhang (张双全), ${ }^{1}$ S. Y. Wang (王守宇), ${ }^{2}$} R. A. Bark, ${ }^{3}$ J. Meng (孟杰), ${ }^{1,4}$ C. G. Wang (王春光),${ }^{1}$ X. G. Wu (吴晓光), ${ }^{5}$ X. Q. Li (李湘庆), ${ }^{1}$ Z. H. Li (李智焕) ${ }^{1}$

S. M. Wyngaardt, ${ }^{4}$ H. L. Zang (藏宏亮), ${ }^{1}$ Z. Q. Chen (陈志强), ${ }^{1}$ H. Y. Wu (吴鸿毅), ${ }^{1}$ F. R. Xu (许甫荣), ${ }^{1}$ Y. L. Ye (叶沿林), ${ }^{1}$ D. X. Jiang (江栋兴), ${ }^{1}$ R. Han (韩芯), ${ }^{1}$ C. G. Li (李晨光), ${ }^{1}$ X. C. Chen (陈潇驰), ${ }^{1}$ Q. Liu (刘强), ${ }^{1}$ J. Feng (冯俊), ${ }^{1}$ B. Yang (杨彪), ${ }^{1}$ Z. H. Li (李宗浩), ${ }^{1}$ S. Wang (王硕), ${ }^{2}$ D. P. Sun (孙大鹏), ${ }^{2}$ C. Liu (刘晨), ${ }^{2}$ Z. Q. Li (李志泉), ${ }^{2}$

N. B. Zhang (张乃波), ${ }^{2}$ R. J. Guo (郭睿巨), ${ }^{2}$ G. S. Li (李广生), ${ }^{5}$ C. Y. He (贺创业), ${ }^{5}$ Y. Zheng (郑云), ${ }^{5}$ C. B. Li (李聪博), ${ }^{5}$ Q. M. Chen (陈启明), ${ }^{5}$ J. Zhong (钟健), ${ }^{5}$ W. K. Zhou (周文奎), ${ }^{5}$ B. J. Zhu (朱宝吉), ${ }^{5}$ L. T. Deng (邓利涛), ${ }^{5}$

M. L. Liu (柳敏良), ${ }^{6}$ J. G. Wang (王建国), ${ }^{6}$ P. Jones, ${ }^{3}$ E. A. Lawrie, ${ }^{3}$ J. J. Lawrie, ${ }^{3}$ J. F. Sharpey-Schafer, ${ }^{3,7}$ M. Wiedeking, ${ }^{3}$ S. N. T. Majola, ${ }^{3,8}$ T. D. Bucher, ${ }^{3,4}$ T. Dinoko, ${ }^{3,7}$ B. Magabuka, ${ }^{3}$ L. Makhathini, ${ }^{3,4}$ L. Mdletshe, ${ }^{3}$ N. A. Khumalo, ${ }^{3,7,9}$ O. Shirinda, ${ }^{3}$ and K. Sowazi ${ }^{3}$

${ }^{1}$ School of Physics and State Key Laboratory of Nuclear Physics and Technology, Peking University, Beijing 100871, China

${ }^{2}$ Shandong Provincial Key Laboratory of Optical Astronomy and Solar-Terrestrial Environment, Institute of Space Sciences, Shandong University, Weihai 264209, China

${ }^{3}$ iThemba LABS, 7129 Somerset West, South Africa

${ }^{4}$ Department of Physics, University of Stellenbosch, Matieland 7602, South Africa

${ }^{5}$ China Institute of Atomic Energy, Beijing 102413, China

${ }^{6}$ Institute of Modern Physics, Chinese Academy of Sciences, Lanzhou 730000, China

${ }^{7}$ Department of Physics, University of the Western Cape, P/B X17 Bellville 7535, South Africa

${ }^{8}$ Department of Physics, University of Cape Town, Rondebosch 7700, South Africa

${ }^{9}$ Department of Physics, University of Zululand, Private Bag X1001, KwaDlangezwa 3886, South Africa

(Received 23 February 2017; revised manuscript received 20 November 2017; published 23 March 2018)

\begin{abstract}
The collective structures of ${ }^{75} \mathrm{Ge}$ have been studied for the first time via the ${ }^{74} \mathrm{Ge}(\alpha, 2 p 1 n){ }^{75} \mathrm{Ge}$ fusionevaporation reaction. Two negative-parity bands and one tentative positive-parity band built on the $v p_{1 / 2}, v f_{5 / 2}$, and $\nu g_{9 / 2}$ states, respectively, are established and compared with the structures in the neighboring $N=43$ isotones. According to the configuration-constrained potential-energy surface calculations, a shape transition from oblate to prolate along the isotopic chain in odd- $A$ Ge isotopes is suggested to occur at ${ }^{75} \mathrm{Ge}$. The properties of the bands in ${ }^{75} \mathrm{Ge}$ are analyzed in comparison with the triaxial particle rotor model calculations.
\end{abstract}

DOI: 10.1103/PhysRevC.97.034322

\section{INTRODUCTION}

As a geometric representation which relates to the fundamental spatial symmetries and deduces different nuclear excitation modes, nuclear shape has long been of great interest in nuclear structure physics. For the even- $A$ Ge isotopes around $A \sim 70$, a gradual shape transition from oblate to prolate along the isotopic chain has been suggested by many experimental studies [1-9]. Meanwhile, the triaxial degree of freedom was found to be significantly involved in these isotopes $[2,4,5,10-14]$ and their neighbors $[15,16]$. Germanium-74 has been suggested to be a crucial nucleus marking the triaxial evolution from soft to rigid [14]. For the odd- $A$ Ge isotopes around $A \sim 70$, the oblate-to-prolate shape transition has been predicted to occur at ${ }^{75} \mathrm{Ge}$ by the finite-range droplet model (FRDM) [17] and the extended Thomas-Fermi-Strutinsky integral (ETFSI) approach [18]. To confirm this shape transition in odd- $A$ Ge isotopes and explore the underlying

\footnotetext{
*chuan@pku.edu.cn

†hhua@pku.edu.cn
}

mechanism, further experimental and theoretical studies are necessary.

In our previous high-spin spectroscopic study [19], the collective structure of ${ }^{73} \mathrm{Ge}$ was expanded significantly. Based on the comparison with similar structures in the neighboring $N=41$ isotones and the cranked Woods-Saxon-Strutinsky calculations, the low-lying states of ${ }^{73} \mathrm{Ge}$ were suggested to have an oblate deformation with a negative $\gamma$ value $\left(\sim-36^{\circ}\right)$. As a neighboring odd- $N$ nucleus of ${ }^{73} \mathrm{Ge}$, the spectroscopy of ${ }^{75} \mathrm{Ge}$ has been previously studied in many experiments [20]. However, since few suitable combinations of stable projectiles and targets are available to populate ${ }^{75} \mathrm{Ge}$ via fusion-evaporation reactions, the collective structure of ${ }^{75} \mathrm{Ge}$ has not been established. Here, we report an experimental investigation on the collective structure of ${ }^{75} \mathrm{Ge}$. The highspin states in ${ }^{75} \mathrm{Ge}$ are populated via the ${ }^{74} \mathrm{Ge}(\alpha, 2 p 1 n){ }^{75} \mathrm{Ge}$ fusion-evaporation reaction. Three rotational bands are established for the first time. They are investigated in terms of the configuration-constrained potential-energy surface (PES) calculations and the triaxial particle rotor model (PRM) calculations. A shape transition from oblate to prolate along the isotopic chain in odd- $A$ Ge isotopes is suggested to occur at 


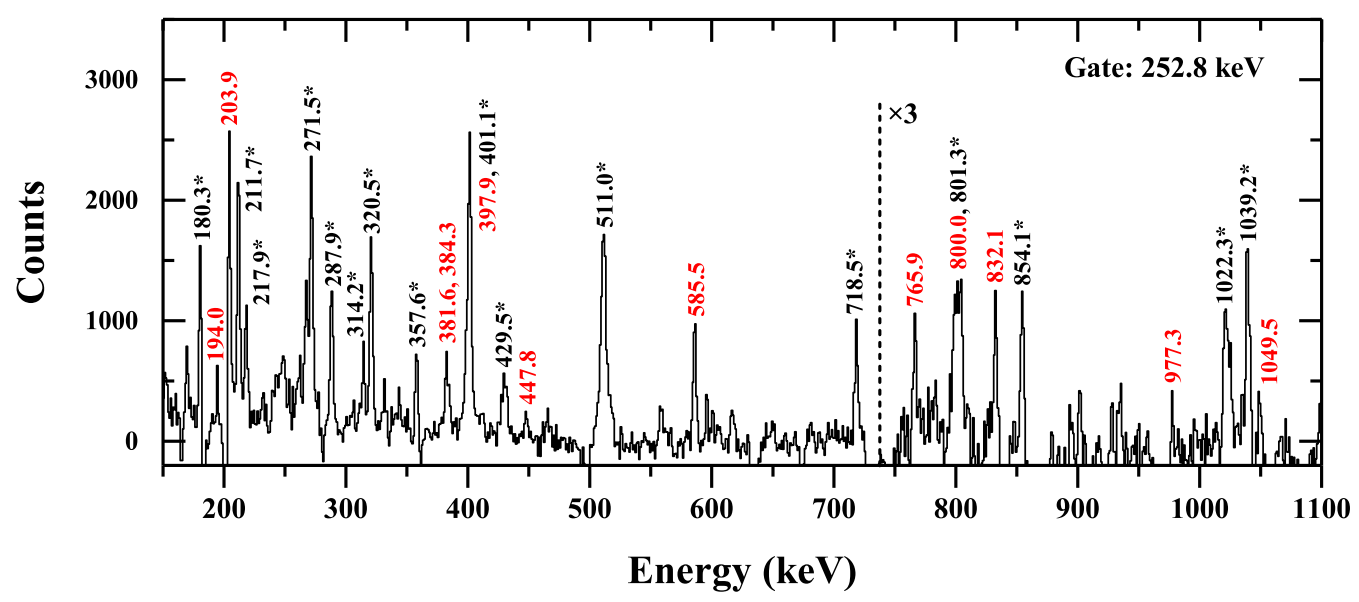

FIG. 1. Coincident $\gamma$-ray spectrum gated on the $252.8-\mathrm{keV}$ transition. For convenience to see, transitions of ${ }^{75} \mathrm{Ge}$ are marked as red. The peaks marked with stars are known contaminants.

${ }^{75} \mathrm{Ge}$, which is consistent with previous FRDM and ETFSI calculations.

\section{EXPERIMENT AND RESULTS}

The experiment was carried out at the Separated Sector Cyclotron of iThemba LABS in South Africa. The ${ }^{74} \mathrm{Ge}(\alpha, x$ pyn $)$ fusion-evaporation reaction with beam energies of 58.6 and 62.6 MeV was used to populate the high-spin states of ${ }^{74} \mathrm{As}$ and neighboring nuclei. The corresponding compound system has a large number of exit channels, and ${ }^{75} \mathrm{Ge}(2 p 1 n)$ is one of a relatively weak exit channel. The target consisted of a $2.85-\mathrm{mg} / \mathrm{cm}^{2}{ }^{74} \mathrm{Ge}$ metallic foil with a $10.8-\mathrm{mg} / \mathrm{cm}^{2}$ carbon backing. In-beam $\gamma$ rays were measured with the AFRODITE array [21], which consisted of eight Compton suppressed clover detectors and two low-energy photon spectrometers at the time of the experiment. The clover detectors were arranged in two rings at $90^{\circ}$ (four clovers) and $135^{\circ}$ (four clovers) with respect to the beam direction. Approximately $1.9 \times 10^{9} \gamma-\gamma$ coincident events were collected with $150 \mathrm{~h}$ of beam time.

A $\gamma-\gamma$ symmetric matrix and a $\gamma-\gamma-\gamma$ cube were built from the coincidence events. For the matrix, a relatively narrow coincident time window of $50 \mathrm{~ns}$ is set to get cleaner coincident spectra, whereas for the cube, a larger coincident time window of $90 \mathrm{~ns}$ is set to get more statistics. The level scheme analysis was performed by using the RADWARE package [22]. In our data analyses, background subtractions were performed with a global fit [23]. To evaluate the background accurately, ROOT software [24] was also used to double-check the background estimation. Typical $\gamma$-ray spectra with single and double gates on the low-lying transitions in ${ }^{75} \mathrm{Ge}$ are shown in Figs. 1 and 2, respectively. With the single gate, as shown in Fig. 1, high statistics can be obtained but with many contaminants. With the double gates, as shown in Fig. 2, the $\gamma$ spectra are clean, and new $\gamma$-ray transitions in ${ }^{75} \mathrm{Ge}$ can be well shown although the statistics are not high. To determine the multipolarities of the $\gamma$-ray transitions, two asymmetric angular distributions from oriented states (ADO) [25] matrices were constructed by using the $\gamma$ rays detected at all angles (the $y$ axis) against those detected at $90^{\circ}$ and $135^{\circ}$ (the $x$ axis), respectively. The multipolarities of the emitted $\gamma$ rays were analyzed by means of the ADO ratio, which was defined as $I_{\gamma}\left(\right.$ at $\left.135^{\circ}\right) / I_{\gamma}\left(\right.$ at $\left.90^{\circ}\right)$. To get accurate ADO values for transitions in ${ }^{75} \mathrm{Ge}$, gates were usually set on the less contaminated transitions. The typical ADO ratios for stretched quadrupole and stretched

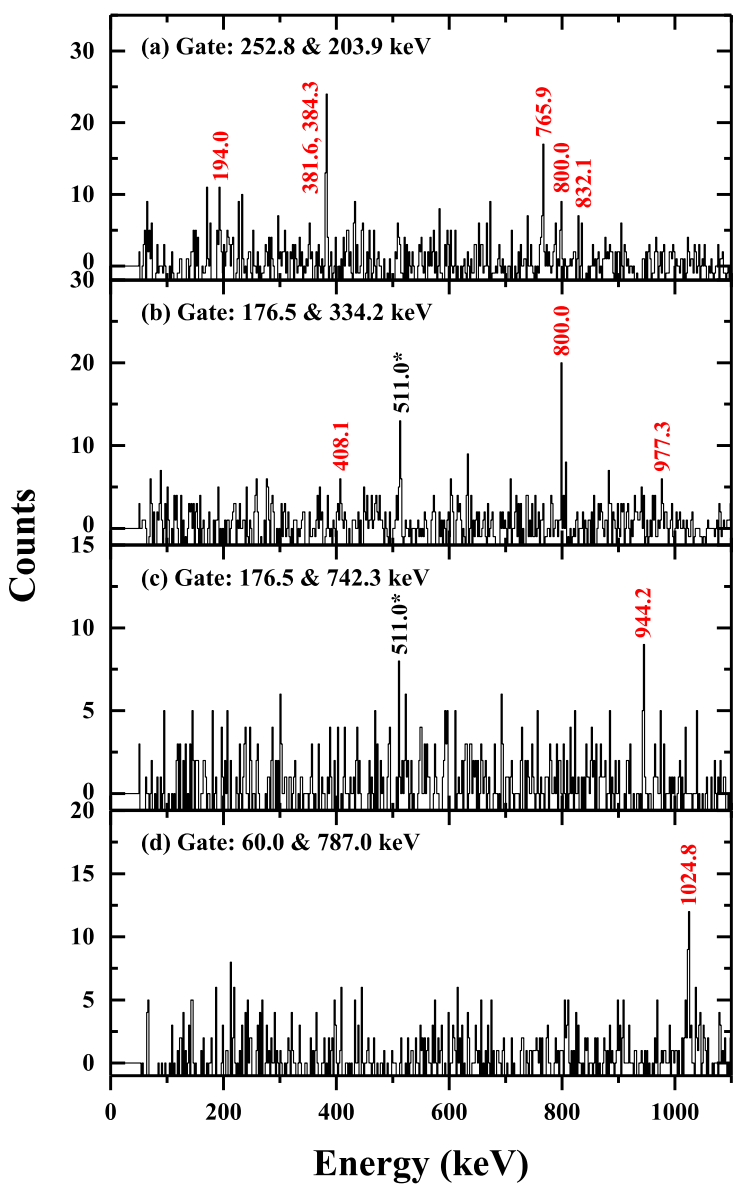

FIG. 2. Coincident $\gamma$-ray spectra double gated on the (a) 252.8and 203.9-keV transitions, (b) 176.5- and 334.2-keV transitions, (c) 176.5- and 742.3-keV transitions, (d) 60.0- and 787.0-keV transitions. The transitions of ${ }^{75} \mathrm{Ge}$ are marked as red. The peaks marked with stars are known contaminants. 


\section{${ }_{32}^{75} \mathrm{Ge}_{43}$}

\section{Band 1}

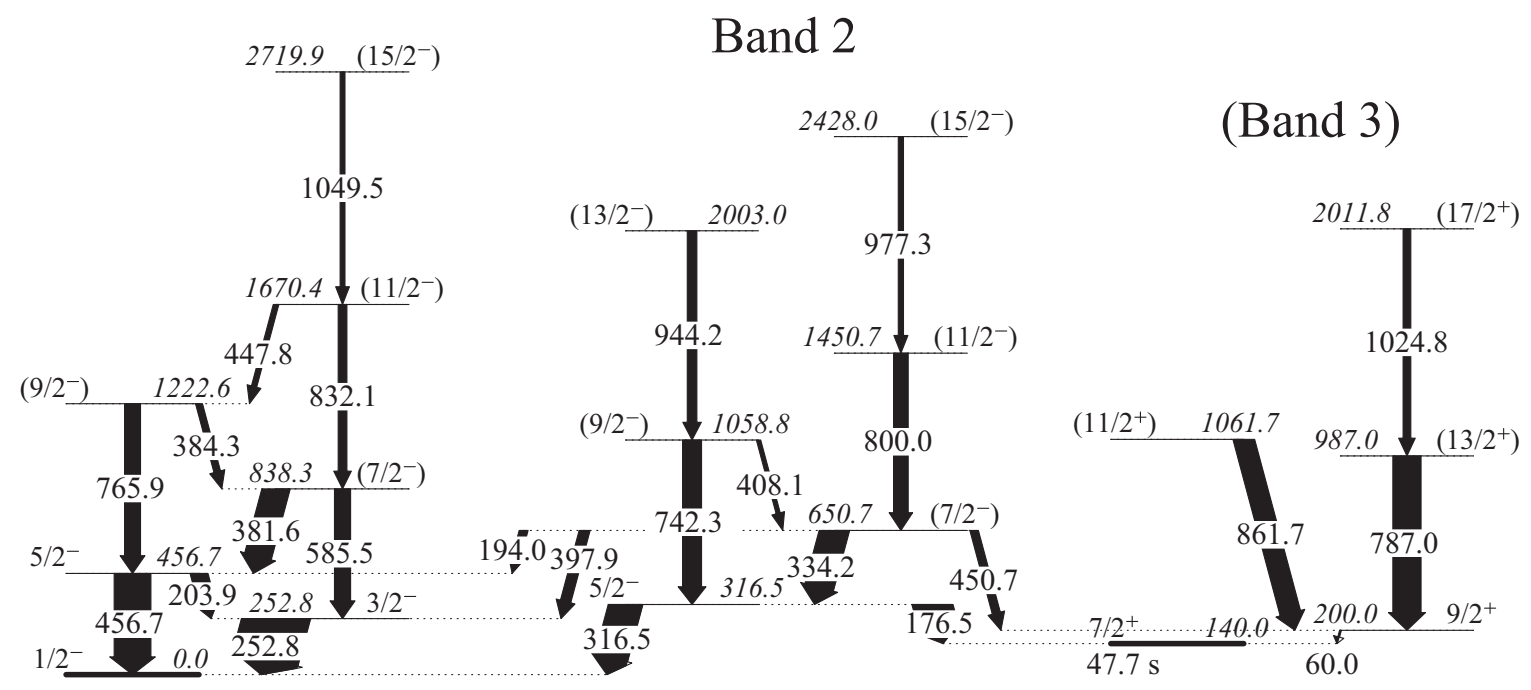

FIG. 3. Partial level scheme of ${ }^{75} \mathrm{Ge}$. The energies are given in $\mathrm{keV}$, and the widths of the arrows are proportional to their relative intensities.

pure dipole transitions were found to be $\sim 1.2$ and $\sim 0.8$, respectively.
A partial level scheme of ${ }^{75} \mathrm{Ge}$ deduced from the present work is shown in Fig. 3. Two negative-parity bands and

TABLE I. $\gamma$-ray energies, excitation energies, relative $\gamma$-ray intensities, ADO ratios, and spin-parity assignments in ${ }^{75}$ Ge.

\begin{tabular}{|c|c|c|c|c|c|}
\hline$E_{\gamma}(\mathrm{keV})$ & $E_{i}(\mathrm{keV})$ & $E_{f}(\mathrm{keV})$ & $I_{\gamma}(\%)$ & $R_{\mathrm{ADO}}$ & $I_{i}^{\pi} \rightarrow I_{f}^{\pi}$ \\
\hline 60.0 & 200.0 & 140.0 & & $0.89(32)$ & $9 / 2^{+} \rightarrow 7 / 2^{+}$ \\
\hline 176.5 & 316.5 & 140.0 & $59.0(138)$ & $0.74(13)$ & $5 / 2^{-} \rightarrow 7 / 2^{+}$ \\
\hline 203.9 & 456.7 & 252.8 & $25.8(57)$ & $0.92(11)$ & $5 / 2^{-} \rightarrow 3 / 2^{-}$ \\
\hline 252.8 & 252.8 & 0.0 & 100.0 & $0.82(10)$ & $3 / 2^{-} \rightarrow 1 / 2^{-}$ \\
\hline 316.5 & 316.5 & 0.0 & $49.9(76)$ & $1.15(10)$ & $5 / 2^{-} \rightarrow 1 / 2^{-}$ \\
\hline 381.6 & 838.3 & 456.7 & $41.2(56)$ & $0.98(11)$ & $\left(7 / 2^{-}\right) \rightarrow 5 / 2^{-}$ \\
\hline 384.3 & 1222.6 & 838.3 & $10.1(27)$ & $0.78(27)$ & $\left(9 / 2^{-}\right) \rightarrow\left(7 / 2^{-}\right)$ \\
\hline 397.9 & 650.7 & 252.8 & $15.9(38)$ & $1.27(19)$ & $\left(7 / 2^{-}\right) \rightarrow 3 / 2^{-}$ \\
\hline 408.1 & 1058.8 & 650.7 & $6.3(25)$ & $0.82(25)$ & $\left(9 / 2^{-}\right) \rightarrow\left(7 / 2^{-}\right)$ \\
\hline 447.8 & 1670.4 & 1222.6 & $8.1(26)$ & $0.80(25)$ & $\left(11 / 2^{-}\right) \rightarrow\left(9 / 2^{-}\right)$ \\
\hline 450.7 & 650.7 & 200.0 & $12.4(35)$ & $0.88(14)$ & $\left(7 / 2^{-}\right) \rightarrow 9 / 2^{+}$ \\
\hline 787.0 & 987.0 & 200.0 & $42.4(131)$ & $1.18(13)$ & $\left(13 / 2^{+}\right) \rightarrow 9 / 2^{+}$ \\
\hline 800.0 & 1450.7 & 650.7 & $22.0(79)$ & $1.13(10)$ & $\left(11 / 2^{-}\right) \rightarrow\left(7 / 2^{-}\right)$ \\
\hline 832.1 & 1670.4 & 838.3 & $13.1(32)$ & $1.29(13)$ & $\left(11 / 2^{-}\right) \rightarrow\left(7 / 2^{-}\right)$ \\
\hline 861.7 & 1061.7 & 200.0 & $31.6(108)$ & $0.99(15)$ & $\left(11 / 2^{+}\right) \rightarrow 9 / 2^{+}$ \\
\hline 944.2 & 2003.0 & 1058.8 & $14.4(45)$ & $1.39(14)$ & $\left(13 / 2^{-}\right) \rightarrow\left(9 / 2^{-}\right)$ \\
\hline 977.3 & 2428.0 & 1450.7 & $7.8(33)$ & $1.11(13)$ & $\left(15 / 2^{-}\right) \rightarrow\left(11 / 2^{-}\right)$ \\
\hline 1024.8 & 2011.8 & 987.0 & $11.6(44)$ & $1.37(18)$ & $\left(17 / 2^{+}\right) \rightarrow\left(13 / 2^{+}\right)$ \\
\hline 1049.5 & 2719.9 & 1670.4 & $7.3(35)$ & $1.31(25)$ & $\left(15 / 2^{-}\right) \rightarrow\left(11 / 2^{-}\right)$ \\
\hline
\end{tabular}


one tentative positive-parity band, which are built on the $1 / 2^{-}, 5 / 2^{-}$, and $7 / 2^{+}$states, respectively, are established for the first time. The level scheme was constructed from the $\gamma-\gamma$ coincidence relationships, intensity balances, and ADO analyses. The results are summarized in Table I. In the present work, the relative intensities of 252.8-, 381.6- and $316.5-\mathrm{keV}$ transitions were extracted from the total projection spectrum. The relative intensities of the other transitions were determined through the single gate spectra and finally normalized to the $252.8-\mathrm{keV}$ transition. The relative intensity of the $60.0-\mathrm{keV}$ transition cannot be extracted from the total projection spectrum accurately due to the large contaminations at the very low-energy region.

For ${ }^{75} \mathrm{Ge}$, the $1 / 2^{-}$ground state has been suggested to have a $p_{1 / 2}$ configuration according to the measured nuclear moments [26]. The levels at 252.8 and $456.7 \mathrm{keV}$ in band 1 have been observed in many previous experiments [20], and $3 / 2^{-}$ and $5 / 2^{-}$were assigned to these two levels, respectively. The present ADO ratio analyses further support the spin and parity assignments of these two levels. As shown in Fig. 2(a) with double gates on the known 252.8- and 203.9-keV transitions in ${ }^{75} \mathrm{Ge}$, new $\gamma$-ray transitions of $381.6,384.3,765.9$, and 832.1 $\mathrm{keV}$ can be seen. By requiring the coincidence with the single $252.8-\mathrm{keV}$ transition, the new coincident $\gamma$-ray transitions of 381.6, 384.3, 447.8, 585.5, 765.9, 832.1, and $1049.5 \mathrm{keV}$ are also observed in Fig. 1. The ADO analyses suggest stretchedquadrupole characteristics for 585.5-, 765.9-, 832.1-, 1049.5$\mathrm{keV}$ transitions and stretched-dipole characteristics for 381.6-, 384.3-, 447.8-keV transitions.

For band 2, its bandhead at $316.5 \mathrm{keV}$ was previously reported with a $5 / 2^{-}$assignment [20], while the level at 650.7 $\mathrm{keV}$ was reported with a tentative $\left(5 / 2^{-}, 7 / 2^{-}\right)$assignment
[20]. According to the ADO value obtained in the current work, the 334.2- and 450.7-keV transitions are suggested to have stretched-dipole characters, and the 397.9-keV transition has stretched-quadrupole character. Therefore, the spin parity of the $650.7-\mathrm{keV}$ level is more likely to be $7 / 2^{-}$. As shown in Figs. 2(b) and 2(c), the new transitions of 408.1, 742.3, 800.0, 944.2 , and $977.3 \mathrm{keV}$ are found to be in coincidence with the low-lying known $\gamma$-ray transitions in ${ }^{75} \mathrm{Ge}$. The ADO analyses suggest stretched-quadrupole characteristics for the 742.3-, 800.0-, 944.2-, and 977.3-keV transitions and stretched-dipole characteristic for the $408.1-\mathrm{keV}$ transition.

For the tentative positive-parity band 3 built on the $7 / 2^{+}$ isomer state, the previously known $9 / 2^{+}$level at $200.0 \mathrm{keV}$ [20] is confirmed here. The levels at 987.0 and $1061.7 \mathrm{keV}$ in band 3 have also been previously reported, and $(5 / 2,7 / 2,9 / 2)^{+}$ and $(5 / 2-13 / 2)^{+}$were tentatively assigned to these two levels, respectively [20]. According to the ADO values obtained in the current work, the 987.0- and 1061.7-keV levels are more likely to be $13 / 2^{+}$and $11 / 2^{+}$states, respectively. As shown in Fig. 2(d), a new transition of $1024.8 \mathrm{keV}$ is found to be in coincidence with the low-lying 60.0- and 787.0-keV transitions, and the ADO analyses suggest a stretched-quadrupole characteristic for this newly observed $1024.8-\mathrm{keV}$ transition.

\section{DISCUSSION}

In the neighboring isotones ${ }^{77} \mathrm{Se}$ [27] and ${ }^{79} \mathrm{Kr}[28,29]$, rotational bands built on $p_{1 / 2}, f_{5 / 2}$, and $g_{9 / 2}$ orbitals have been well established. These bands are compared with those of ${ }^{75} \mathrm{Ge}$ in Fig. 4. The similarity visible in Fig. 4 indicates that band 2 is most likely built on the $f_{5 / 2}$ orbital and band 3 has the $g_{9 / 2}$ configuration like those in ${ }^{77} \mathrm{Se}$ and ${ }^{79} \mathrm{Kr}$. In addition,

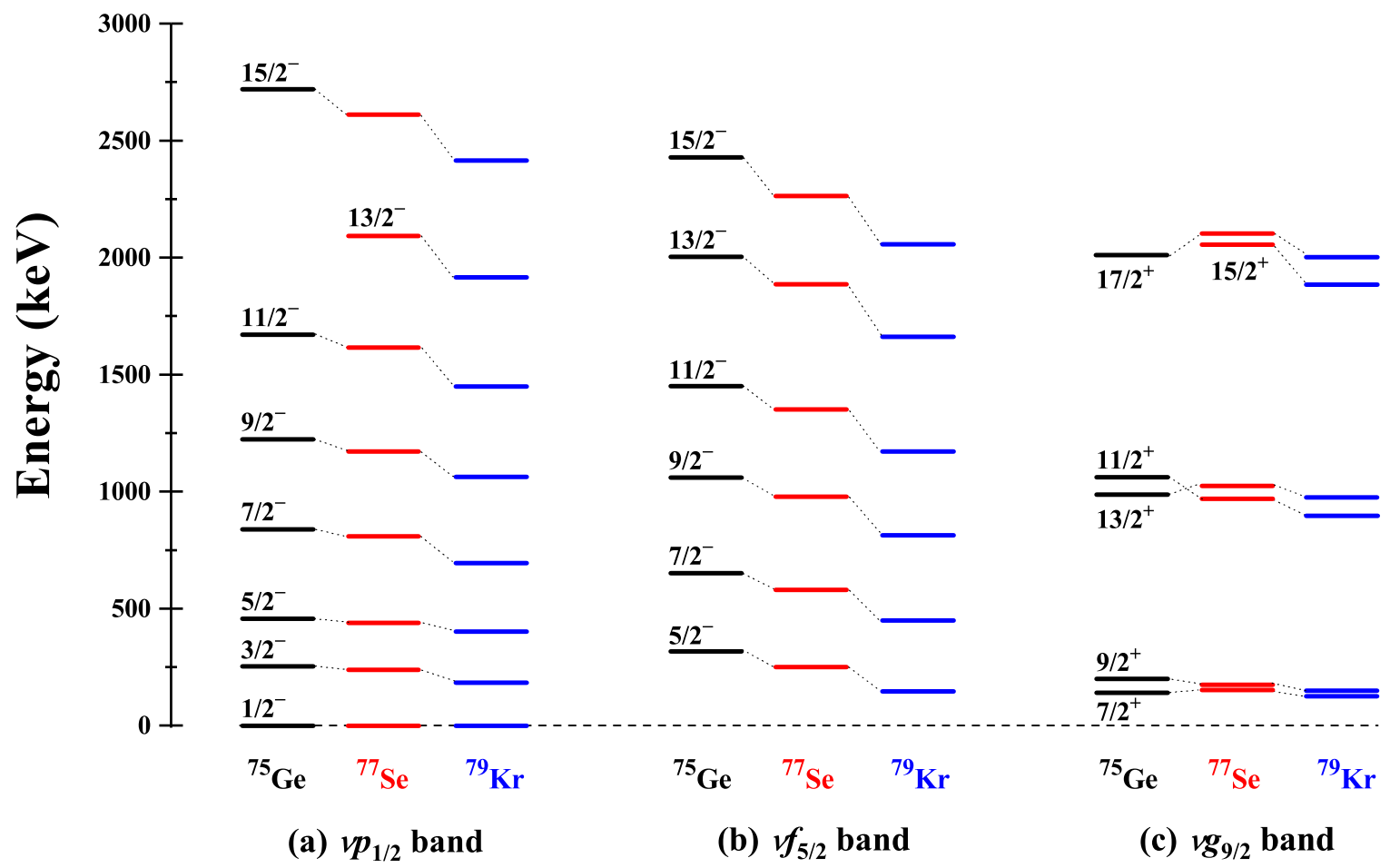

FIG. 4. Comparison of the $v p_{1 / 2}, v f_{5 / 2}$, and $v g_{9 / 2}$ bands in isotones ${ }^{75} \mathrm{Ge},{ }^{77} \mathrm{Se}[27]$, and ${ }^{79} \mathrm{Kr}$ [29]. 
one interesting feature in Fig. 4 is that the level position of favored and unfavored signature partners in the $v g_{9 / 2}$ band of ${ }^{75} \mathrm{Ge}$ reverses around spin $11 / 2 \hbar$, which is not observed in the heavier $N=43$ isotones and reveals the structural change between ${ }^{75} \mathrm{Ge}$ and heavier $N=43$ isotones.

As mentioned in the Introduction, the shape of the odd- $A$ $\mathrm{Ge}$ isotopes evolves from oblate to prolate as a function of neutron number. The nucleus ${ }^{75} \mathrm{Ge}$ is predicted to be the crucial nucleus where the shape changes from oblate to prolate by the FRDM [17] and the ETFSI approach [18]. For ${ }^{73} \mathrm{Ge}$ and ${ }^{75} \mathrm{Ge}$, their ground states have been found to be built on different orbitals: ${ }^{73} \mathrm{Ge}$ on the $g_{9 / 2}$ orbital and ${ }^{75} \mathrm{Ge}$ on the $p_{1 / 2}$ orbital. The different polarization effects of the $g_{9 / 2}$ and $p_{1 / 2}$ orbitals might be the underlying reason for the oblate-to-prolate shape transition. To get a detailed understanding of the shape transition in these odd- $A$ isotopes, the configuration-constrained PESs [30] for ${ }^{73} \mathrm{Ge}$ and ${ }^{75} \mathrm{Ge}$ have been calculated. The calculated PESs on the $\beta-\gamma$ plane for ${ }^{73} \mathrm{Ge}$ and ${ }^{75} \mathrm{Ge}$ are illustrated in Fig. 5. It can be seen that the $9 / 2^{+}$ground state of ${ }^{73} \mathrm{Ge}$ has an oblate deformation $\left(\beta_{2}=0.23, \gamma=-41^{\circ}\right)$, whereas the $1 / 2^{-}$ground state of ${ }^{75} \mathrm{Ge}$ has a prolate deformation $\left(\beta_{2}=0.17, \gamma=-19^{\circ}\right)$. The PES results suggest a shape transition from oblate to prolate occurs at ${ }^{75} \mathrm{Ge}$, which is consistent with the previous theoretical predictions $[17,18]$. In addition, one interesting feature is that,

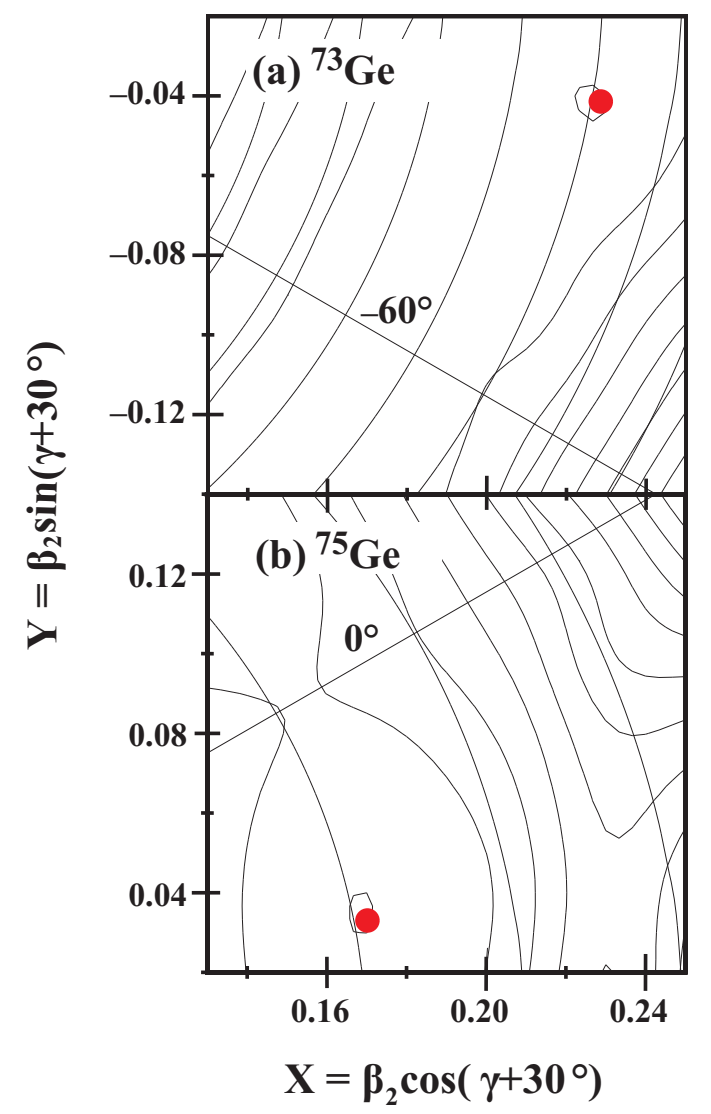

FIG. 5. The calculated PESs for ground states in (a) ${ }^{73} \mathrm{Ge}$ and (b) ${ }^{75} \mathrm{Ge}$. The energy contours are at $100-\mathrm{keV}$ intervals. The corresponding minima are $\left(\beta_{2}=0.23, \gamma=-41^{\circ}\right),\left(\beta_{2}=0.17, \gamma=-19^{\circ}\right)$. with the change in occupied orbital from $g_{9 / 2}$ to $p_{1 / 2}$, the quadrupole deformation decreases from 0.23 to 0.17 .

To further study the properties of the bands in ${ }^{75} \mathrm{Ge}$, the triaxial PRM calculations [31] have been performed. A detailed description of the PRM can be found Refs. [31-34]. The values of $\kappa$ and $\mu$ for the valance neutron in the Nilsson-type Hamiltonian are taken from Ref. [35], i.e., $\kappa=0.090$ and $\mu=0.25$ for the main oscillator quantum number $N=3$, and $\kappa=0.070$ and $\mu=0.39$ for $N=4$. The deformation parameters $\left(\beta_{2}, \gamma\right)$ are adopted from configuration-constrained PES calculations, i.e., $\left(0.27,-35^{\circ}\right)$ for the $\nu g_{9 / 2}$ band, $\left(0.17,-19^{\circ}\right)$ for the $v p_{1 / 2}$ band, and $\left(0.17,-22^{\circ}\right)$ for the $v f_{5 / 2}$ band. The hexadecapole deformation is neglected in the present PRM investigation. The neutron Fermi energy $\lambda_{n}$ is taken to be the energy of the single-particle level occupied by the valence neutron, and the pairing gap $\Delta$ is determined by the experimental odd-even mass difference. The Coriolis attenuation parameter $\xi=0.6$ is used.

The PRM-calculated energy spectra for the positiveand negative-parity bands in ${ }^{75} \mathrm{Ge}$ are compared with the

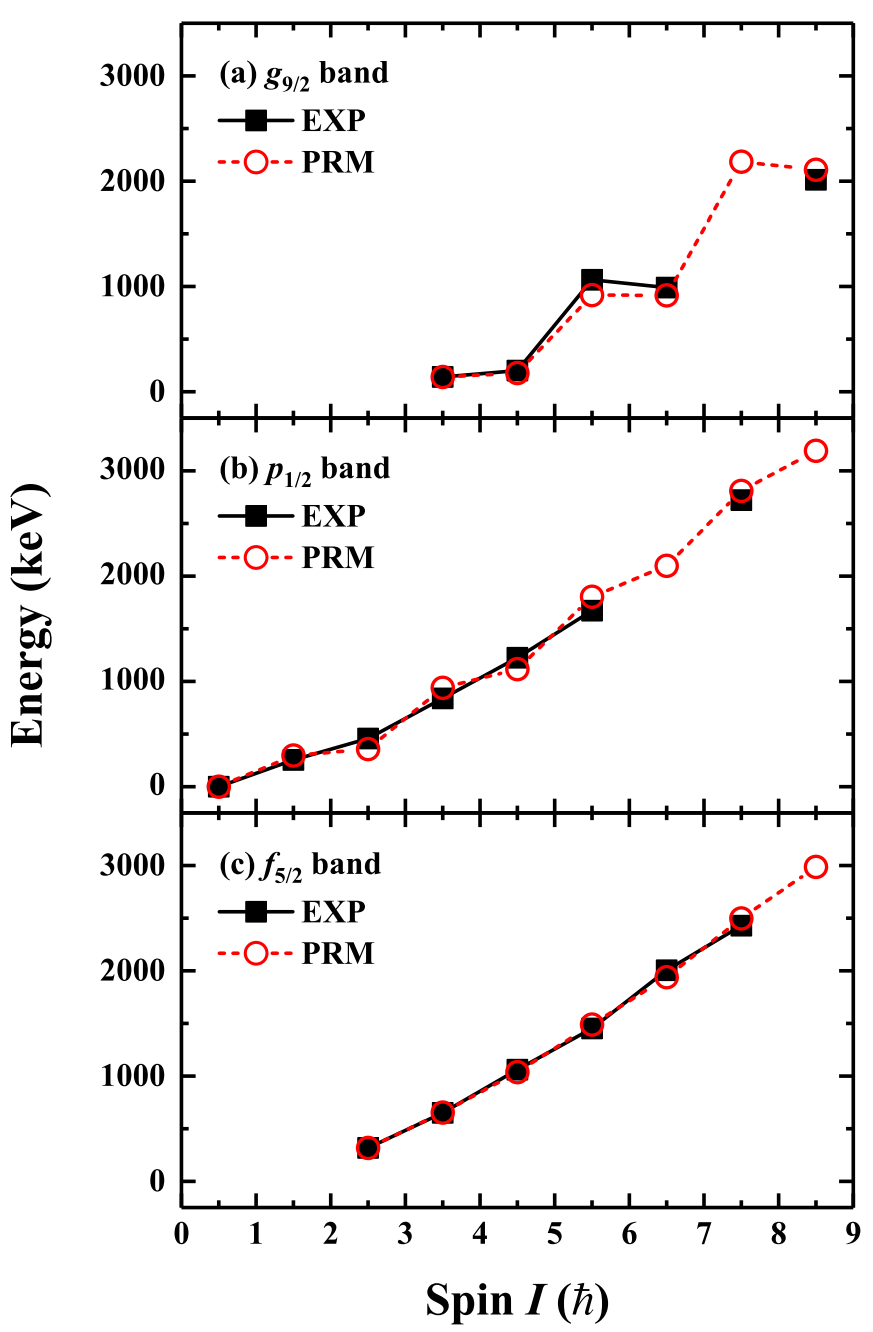

FIG. 6. The calculated energy spectra $E(I)$ for the (a) $v g_{9 / 2}$, (b) $v p_{1 / 2}$, and (c) $v f_{5 / 2}$ bands in ${ }^{75} \mathrm{Ge}$ by the PRM in comparison with the experimental data. 
experimental data in Fig. 6. It can be seen that the triaxial PRM can well reproduce the experimental excitation energies of $v g_{9 / 2}, v p_{1 / 2}$, and $v f_{5 / 2}$ bands in ${ }^{75} \mathrm{Ge}$. In particular, the signature splitting of the $g_{9 / 2}$ band is well reproduced. The good agreement between the PRM calculations and the experimental data further support the configuration assignments for these bands in ${ }^{75} \mathrm{Ge}$. According to the PRM calculations, the configuration of the positive-parity band (band 3 ) is almost pure $\nu g_{9 / 2}$, whereas in the negative-parity bands (bands 1 and 2) there exists strong mixing of the $v p_{1 / 2}$ and $v f_{5 / 2}$ configurations.

Although the present experimental collective structures of ${ }^{75} \mathrm{Ge}$ have not been extended to the band crossing region, theoretical studies can give some hints on the characters of band crossings in the yrast negative-parity $v f_{5 / 2}$ band and the positive-parity $\nu g_{9 / 2}$ band. For the negative-parity $v f_{5 / 2}$ band of ${ }^{75} \mathrm{Ge}$, according to the cranked Woods-SaxonStrutinsky calculations by means of total-Routhian-surface (TRS) methods [36,37], the first band crossing is caused by the $g_{9 / 2}$ neutron alignment and occurs at a rotational frequency of $0.40-0.50 \mathrm{MeV}$. Meanwhile, the $g_{9 / 2}$ proton alignment is predicted to occur at a much higher rotational frequency of $0.90-1.00 \mathrm{MeV}$, which is almost simultaneous with the second $g_{9 / 2}$ neutron alignment. For the positive-parity $v g_{9 / 2}$ band in ${ }^{75} \mathrm{Ge}$, due to the effect of Pauli blocking by the occupation of the neutron $g_{9 / 2}$ orbital, TRS calculations predict that the first $g_{9 / 2}$ neutron alignment shifts to a higher rotational frequency of $0.65-0.75 \mathrm{MeV}$. Meanwhile, the first $g_{9 / 2}$ proton alignment does not occur up to a rotational frequency $1.00 \mathrm{MeV}$.

\section{SUMMARY}

The collective structures of ${ }^{75} \mathrm{Ge}$ have been studied for the first time via the ${ }^{74} \mathrm{Ge}(\alpha, 2 p 1 n){ }^{75} \mathrm{Ge}$ fusion-evaporation reaction. Two negative-parity bands and one tentative positiveparity band built on the $v p_{1 / 2}, v f_{5 / 2}$, and $v g_{9 / 2}$ states, respectively, are newly established and compared with the structures in the neighboring $N=43$ isotones. For the odd- $A$ Ge isotopes, the shape transition from oblate to prolate along the isotopic chain is suggested to occur at ${ }^{75} \mathrm{Ge}$, which is consistent with the previous theoretical predictions. The properties of new bands in ${ }^{75} \mathrm{Ge}$ are analyzed in comparison with the triaxial PRM calculations. The band crossings induced by the $g_{9 / 2}$ particle alignment in ${ }^{75} \mathrm{Ge}$ are studied in terms of cranked Woods-Saxon-Strutinsky calculations. Further confirmation of the band crossings calls for more experimental studies.

\section{ACKNOWLEDGMENTS}

This work was supported by the Natural Science Foundation of China under Grants No. 11675003, No. 11175003, No. 11235001, No. 11320101004, No. 11375015, No. 11461141002, No. 11461141001, and No. J1103206, the Chinese Major State Basic Research Development Program under Grant No. 2013CB834400, the Natural Research Foundation of South Africa Grant No. 92791. The authors wish to thank Dr. Q. W. Fan for making the target and the staff of iThemba LABS and HI-13 for their support.
[1] E. F. Aguilera, J. J. Vega, J. J. Kolata, A. Morsad, R. G. Tighe, and X. J. Kong, Phys. Rev. C 41, 910 (1990).

[2] F. Ballester, E. Casal, and J. B. A. England, Nucl. Phys. A 490, 227 (1988).

[3] R. Lecomte, G. Kajrys, S. Landsberger, P. Paradis, and S. Monaro, Phys. Rev. C 25, 2812 (1982).

[4] B. Kotliński et al., Nucl. Phys. A 519, 646 (1990).

[5] Y. Toh et al., Eur. Phys. J. A 9, 353 (2000).

[6] Y. Toh et al., J. Phys. G 27, 1475 (2001).

[7] R. Lecomte, M. Irshad, S. Landsberger, P. Paradis, and S. Monaro, Phys. Rev. C 22, 1530 (1980).

[8] R. Lecomte, M. Irshad, S. Landsberger, G. Kajrys, P. Paradis, and S. Monaro, Phys. Rev. C 22, 2420 (1980).

[9] M. Sugawara et al., Eur. Phys. J. A 16, 409 (2003).

[10] W. Andrejtscheff and P. Petkov, Phys. Lett. B 329, 1 (1994).

[11] W.-T. Chou, D. S. Brenner, R. F. Casten, and R. L. Gill, Phys. Rev. C 47, 157 (1993).

[12] S. F. Shen, S. J. Zheng, F. R. Xu, and R. Wyss, Phys. Rev. C 84, 044315 (2011).

[13] Y. Toh et al., Phys. Rev. C 87, 041304(R) (2013).

[14] J. J. Sun et al., Phys. Lett. B 734, 308 (2014).

[15] S. Y. Wang et al., Phys. Lett. B 703, 40 (2011).

[16] C. Liu et al., Phys. Rev. Lett. 116, 112501 (2016).

[17] P. Möller, A. J. Sierk, T. Ichikawa, and H. Sagawa, At. Data Nucl. Data Tables 109-110, 1 (2016).

[18] Y. Aboussir, J. M. Pearson, A. K. Dutta, and F. Tondeur, At. Data Nucl. Data Tables 61, 127 (1995).

[19] J. J. Sun et al., Phys. Rev. C 92, 054302 (2015).

[20] A. Negret and B. Singh, Nucl. Data Sheets 114, 841 (2013), and references therein.
[21] J. Sharpey-Schafer, Nucl. Phys. News 14, 5 (2004).

[22] D. C. Radford, Nucl. Instrum. Methods Phys. Res., Sect. A 361, 297 (1995).

[23] D. C. Radford, Nucl. Instrum. Methods Phys. Res., Sect. A 361, 306 (1995).

[24] https://root.cern.ch/.

[25] M. Piiparinen et al., Nucl. Phys. A 605, 191 (1996).

[26] A. F. Oluwole, S. G. Schmelling, and H. A. Shugart, Phys. Rev. C 2, 228 (1970).

[27] G. D. Johns, J. Döring, R. A. Kaye, G. N. Sylvan, and S. L. Tabor, Phys. Rev. C 55, 660 (1997).

[28] R. Schwengner et al., Nucl. Phys. A 509, 550 (1990).

[29] G. D. Johns, J. Döring, J. W. Holcomb, T. D. Johnson, M. A. Riley, G. N. Sylvan, P. C. Womble, V. A. Wood, and S. L. Tabor, Phys. Rev. C 50, 2786 (1994).

[30] F. R. Xu, P. M. Walker, J. A. Sheikh, and R. Wyss, Phys. Lett. B 435, 257 (1998).

[31] B. Qi, S. Q. Zhang, S. Y. Wang, and J. Meng, Int. J. Mod. Phys. E 18, 109 (2009).

[32] S. Q. Zhang, B. Qi, S. Y. Wang, and J. Meng, Phys. Rev. C 75, 044307 (2007).

[33] S. E. Larsson, G. Leander, and I. Ragnarsson, Nucl. Phys. A 307, 189 (1978).

[34] I. Ragnarsson and P. B. Semmes, Hyperfine Interact. 43, 423 (1988).

[35] T. Bengtsson and I. Ragnarsson, Nucl. Phys. A 436, 14 (1985).

[36] W. Satuła, R. Wyss, and P. Magierski, Nucl. Phys. A 578, 45 (1994).

[37] F. R. Xu, W. Satuła, and R. Wyss, Nucl. Phys. A 669, 119 (2000). 